\title{
Rickettsia japonica Infections in Humans, Xinyang, China, 2014-2017
}

\author{
Hao Li, Pan-He Zhang, Juan Du, \\ Zhen-Dong Yang, Ning Cui, Bo Xing, \\ Xiao-Ai Zhang, Wei Liu
}

During 2014-2017, we screened for Rickettsia japonica infection in Xinyang, China, and identified 20 cases. The major clinical manifestations of monoinfection were fever, asthenia, myalgia, rash, and anorexia; laboratory findings included thrombocytopenia and elevated hepatic aminotransferase concentrations. Physicians in China should consider $R$. japonica infection in at-risk patients.

$\mathrm{E}$ merging and reemerging spotted fever group (SFG) rickettsioses are increasingly spreading and being recognized worldwide. Japanese spotted fever (JSF), caused by Rickettsia japonica and first reported in Japan in 1984 $(1,2)$, is an SFG rickettsiosis characterized by fever, malaise, chills, headache, rash, and eschars $(3,4)$. JSF cases have been increasingly reported, but mostly in Japan and the nearby countries of South Korea, the Philippines, and Thailand (4-7). R. japonica has been detected in 3 genera (Haemaphysalis, Dermacentor, Ixodes) and 8 species of ticks $(8,9)$. Because of the wide spectrum of ticks in which this bacterium has been found, $R$. japonica is postulated to be distributed more widely throughout China. The identification of $R$. japonica in $H$. flava and $H$. hystricis ticks in Wuhan, China, further corroborated this hypothesis (10). Also, serologic responses to $R$. japonica were detected in patients in Hainan Province in southern China $\approx 3$ decades ago (11). However, laboratory-confirmed JSF cases have been identified in only a few patients in China $(12,13)$. In this study, we aimed to determine if more JSF cases were occurring in China by using hospital-based surveillance.

\section{The Study}

During March 2014-June 2017, we screened 2,236 febrile patients seeking treatment at The People's Liberation Army 990 Hospital in Xinyang, Henan Province, eastern central China (Appendix Figure, https://wwwnc.cdc.gov/

Author affiliations: Beijing Institute of Microbiology and

Epidemiology, Beijing, China (H. Li, P.-H. Zhang, B. Xing,

X.-A. Zhang, W. Liu); Peking University, Beijing (J. Du); People's

Liberation Army 990 Hospital, Xinyang, China (Z.-D. Yang, N. Cui)

DOI: https://doi.org/10.3201/eid2509.171421
EID/article/25/9/17-1421-App1.pdf) with a history of tick bite or field activity within the previous month for infection with $R$. japonica. We extracted DNA from peripheral blood samples collected during the acute phase of illness and screened them by a nested PCR concurrently targeting the citrate synthase gene $(g l t \mathrm{~A}), 17-\mathrm{kDa}$ antigen-encoding gene (htrA), and outer membrane protein A-encoding gene (ompA), as previously described (14). To minimize the risk for contamination, we performed template isolation and PCR setup in separate rooms with specified pipette sets, used certified DNAase- and RNase-free filter barrier tips to prevent aerosol contamination, and performed all PCR assays with appropriate controls. After amplification and sequencing, we found that 20 patients were infected with R. japonica.

The glt A gene sequence (456-bp) obtained from all 20 samples (GenBank accession no. MF693145) showed $100 \%$ similarity to the corresponding gene of $R$. japonica strain YH (GenBank accession no. AP017602). The htrA gene sequence (394-bp) obtained from 18 samples (GenBank accession no. MF693146) was identical to that of $R$. japonica $\mathrm{YH}$, and the sequence in the remaining 2 samples (GenBank accession no. MF693147) differed by only 3 bp. The ompA gene sequence (311-bp) found in 16 samples (GenBank accession no. MF693148) was identical to that of $R$. japonica $\mathrm{YH}$, and the remaining 4 samples each differed by just 1 bp (GenBank accession nos. MF693149-52) (Figure 1).

We also assessed other tickborne agents, including Anaplasma phygocytophilum, Anaplasma capra, Ehrlichia chaffeensis, Borrelia burgdorferi sensu lato, Babesia microti, and severe fever with thrombocytopenia syndrome virus, by PCR or reverse transcription PCR. Six of 20 patients were co-infected with severe fever with thrombocytopenia syndrome virus, so we excluded them from clinical analysis.

We tested the acute serum samples collected $\leq 7$ days after illness onset and the convalescence serum samples collected $\geq 14$ days after illness onset from the 14 patients with $R$. japonica monoinfection in parallel using an indirect immunofluorescence assay for Rickettsia heilongjiangensis IgG. We considered an IgG titer $\geq 1: 64$ a positive reaction. Using this definition, we found that 6 patients had a seroconversion, and the other 8 had a 4-fold increased IgG titer; thus, all patients had an acute infection with SFG rickettsiae. 


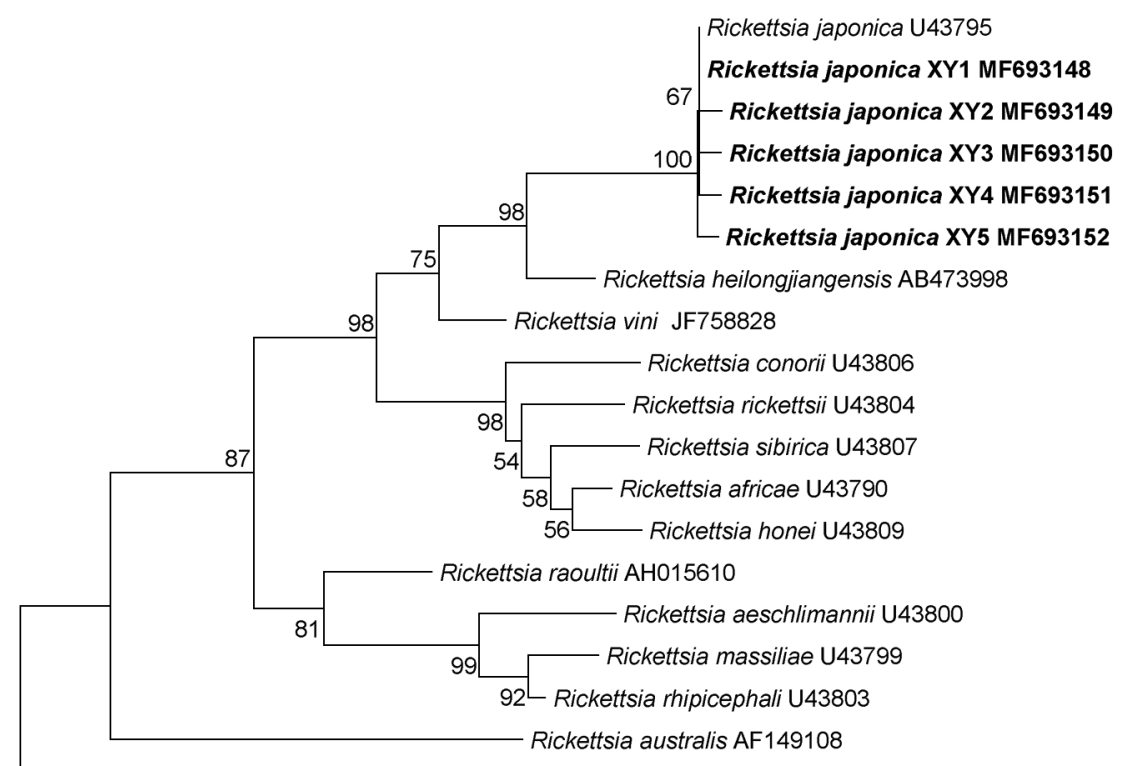

Candidatus Rickettsia tarasevichiae KF008247
Figure 1. Phylogenetic analysis of Rickettsia species from febrile patients treated at The People's Liberation Army 990 Hospital for Rickettsia japonica infection, Xinyang, China, March 2014June 2017 (bold), and reference species. Tree was constructed on the basis of the outer member protein A nucleotide (311-bp) gene sequence. We used the maximum-likelihood method with the best substitution model (Tamura 3-parameter plus gamma) and MEGA version 5.0 (http://www.megasoftware. net). We applied a bootstrap analysis of 1,000 replicates to assess the reliability of the reconstructed phylogenies. GenBank accession numbers are provided. Scale bar indicates estimated evolutionary distance.
All 14 patients had a history of field activity within the previous month, and 5 had a history of tick bite. Median patient age was 61.5 (range 44-77) years; 9 were men. The median time from tick bite to onset of illness was 4 (range 3-8) days and from onset of illness to physician visit 5 (range 2-7) days. The median duration of hospitalization was 6 (range 4-10) days.

Fever and asthenia were reported by all 14 patients. Other nonspecific symptoms included myalgia (10/14), lymphadenopathy (4/14), headache (3/14), dizziness (3/14), and chills (2/14). Gastrointestinal manifestations included anorexia $(9 / 14)$, nausea (7/14), vomiting (3/14), and diarrhea (1/14). Three patients had cough and pneumonia, and we observed rash in 10 patients (Figure 2). The median time from onset of illness to rash was 4 (range 3-5) days, and the median duration of rash was 5.5 (range 4-8) days. Only 3 patients had an eschar. Other signs included splenomegaly (2/14) and facial edema (1/14).

Urinalysis on admission revealed microscopic hematuria in 2 patients and a slight or moderate proteinuria in 8 patients. The most common findings on laboratory tests were thrombocytopenia, elevated hepatic aminotransferase concentrations, elevated serum lactate dehydrogenase, and hypoalbuminemia, followed by hyponatremia, anemia, hyperbilirubinemia, hypopotassemia, leukopenia, and elevated serum creatine kinase (Table). Mild multiple organ dysfunction developed in 3 patients.

Because of the appearance of typical rash, we suspected SFG rickettsioses in 10 patients and treated them with doxycycline (100 mg $2 \times / \mathrm{d})$ until their fevers dissipated and clinical disease improved (median 4 [range 3-5] days). The other 4 patients were treated with the antimicrobial drugs
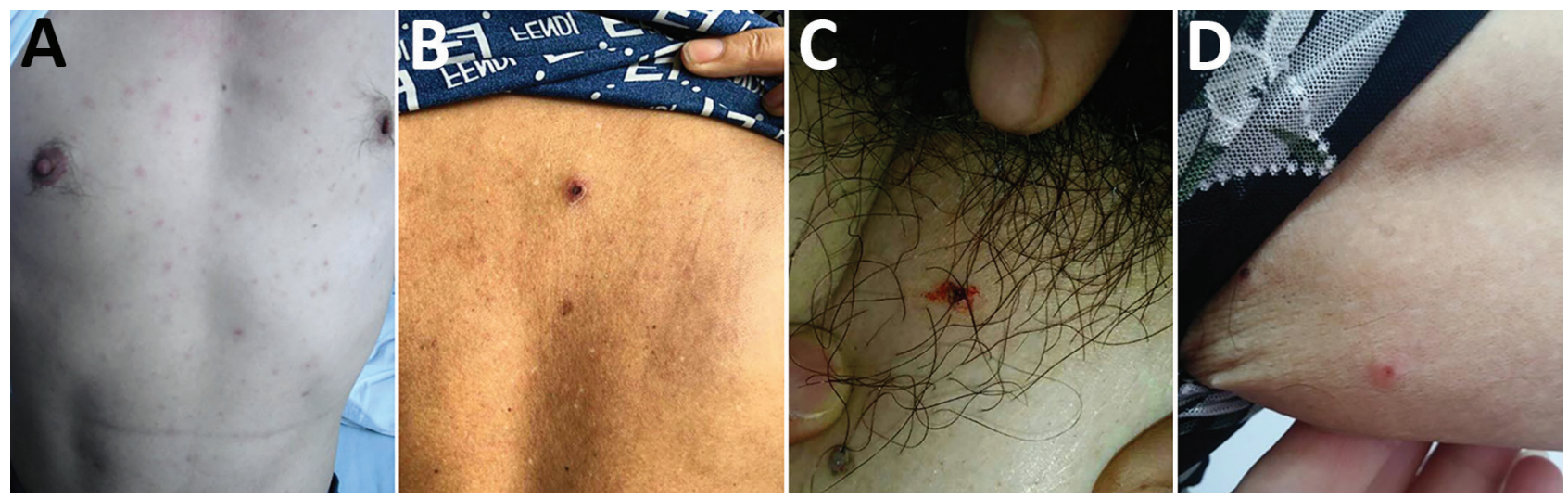

Figure 2. Lesions on patients with Rickettsia japonica infection, Xinyang, China, March 2014-June 2017. A) Rash on ventrum; B) rash and eschar on back; C) eschar on femoribus internus; D) tick bite site on left armpit. 
Table. Laboratory test results of samples from 14 patients with Rickettsia japonica infection at different time points, China, 2014-2017*

\begin{tabular}{lccc}
\hline & \multicolumn{2}{c}{ No. patients } \\
\cline { 2 - 4 } Result & At admission & During hospitalization & At discharge from hospital \\
\hline Anemia, $<3.5 \times 10^{12}$ erythrocytes/L & 0 & 5 & 3 \\
Leukopenia, $<4.0 \times 10^{9}$ leukocytes/L & 3 & 3 & 0 \\
Thrombocytopenia, $<150 \times 10^{9}$ platelets/L & 9 & 11 & 0 \\
Hyperbilirubinemia, albumin $>17.1 \mu \mathrm{mol} / \mathrm{L}$ & 4 & 5 & 2 \\
Hypoalbuminemia, albumin $<35.0 \mathrm{~g} / \mathrm{L}$ & 3 & 9 & 7 \\
Hyponatremia, sodium $<135.0 \mathrm{mmol} / \mathrm{L}$ & 4 & 6 & 2 \\
Hypopotassemia, potassium $<3.5 \mathrm{mmol} / \mathrm{L}$ & 2 & 4 & 2 \\
Increased ALT level, $>0.67 \mu \mathrm{kat} / \mathrm{L}$ & 8 & 10 & 5 \\
Increased AST level, $>0.67 \mu \mathrm{kat} / \mathrm{L}$ & 10 & 12 & 5 \\
Increased LDH level, $>4.1 \mu \mathrm{kat} / \mathrm{L}$ & 10 & 11 & 7 \\
Increased CK level, $>4.1 \mu \mathrm{kat} / \mathrm{L}$ & 4 & 3 & \\
\hline${ }^{*}$ ALT, alanine aminotransferase; AST, aspartate aminotransferase; $\mathrm{CK}$, creatine kinase; $\mathrm{LDH}$, lactate dehydrogenase. & \\
\hline
\end{tabular}

cefminox or cefoperazone for a median of 6 (range 5-7) days. By the time patients were discharged from the hospital, their leukocyte and platelet counts had returned to reference range levels, but half of the patients still had laboratory test values outside of their respective reference ranges (Table). No patients reported clinically significant sequelae at their 2-week follow-up appointment.

\section{Conclusions}

The tick vectors of $R$. japonica (i.e., H. flava, H. hystricis, $H$. cornigera, H. longicornis, I. ovatus) are widely distributed throughout China (15), providing $R$. japonica ample opportunity to infect humans. By applying molecular screening techniques to simultaneously amplify 3 genes, we identified $R$. japonica infection in 14 patients.

The clinical signs and symptoms of $R$. japonica infection in our patient cohort differed from those reported in patients in Japan $(3,4)$. We frequently observed fever, asthenia, and rash but not chills and headache. We also saw fewer eschars in our patient cohort, potentially because they were underreported during clinical examination; eschars are not always easy to identify. In addition, the patients we identified usually had myalgia and gastrointestinal symptoms.

These clinical findings expand the available knowledge of the disease spectrum of $R$. japonica infection. Compared with endemic rickettsiosis caused by Candidatus Rickettsia tarasevichiae infection (reference 16 in Appendix), rash is commonly seen, and hemorrhagic and neurologic signs and symptoms are rarely seen in patients with $R$. japonica infection. These distinctive features could be used to make a differential diagnosis.

We frequently observed in our patient cohort thrombocytopenia, hypoalbuminemia, elevated hepatic enzyme activity, and elevated lactate dehydrogenase levels, findings resembling those of patients with common SFG rickettsioses (reference 17 in Appendix). In general, disease is mild or moderate, and no deaths have been recorded, although mild multiple organ dysfunction developed in several patients.
In conclusion, we identified $R$. japonica as an emerging tickborne pathogen in China. Physicians in areas where $H$. longicornis and other competent vectors for $R$. japonica are endemic should be aware of the risk for infection in humans and prescribe doxycycline to patients in cases of ineffective therapy with other antimicrobial drugs. Surveillance needs to be extended to improve our understanding of the health burden of JSF.

This study was supported by the National Natural Science Foundation of China $(81825019,81722041,81472005$, and 81621005), the China Mega-Project for Infectious Diseases (2018ZX10713002-002), the National Key Research and Development Program of China (2016YFX1201905), and the New Star Plan of Science and Technology of Beijing (Z171100001117089).

\section{About the Author}

Dr. Li is a scientist in the State Key Laboratory of Pathogen and Biosecurity, Beijing Institute of Microbiology and Epidemiology, Beijing, China. His research interests include microbiology, epidemiology, and ecology of tickborne diseases.

\section{References}

1. Mahara F, Koga K, Sawada S, Taniguchi T, Shigemi F, Suto T, et al. The first report of the rickettsial infections of spotted fever group in Japan: three clinical cases [in Japanese]. Kansenshogaku Zasshi. 1985;59:1165-72. https://doi.org/10.11150/ kansenshogakuzasshi1970.59.1165

2. Uchida T, Uchiyama T, Kumano K, Walker DH. Rickettsia japonica sp. nov., the etiological agent of spotted fever group rickettsiosis in Japan. Int J Syst Bacteriol. 1992;42:303-5. https://doi.org/10.1099/00207713-42-2-303

3. Mahara F. Japanese spotted fever: report of 31 cases and review of the literature. Emerg Infect Dis. 1997;3:105-11. https://doi.org/ 10.3201/eid0302.970203

4. Mahara F. Rickettsioses in Japan and the Far East. Ann N Y Acad Sci. 2006;1078:60-73. https://doi.org/10.1196/annals.1374.007

5. Camer GA, Alejandria M, Amor M, Satoh H, Muramatsu Y, Ueno $\mathrm{H}$, et al. Detection of antibodies against spotted fever group Rickettsia (SFGR), typhus group Rickettsia (TGR), and Coxiella burnetii in human febrile patients in the Philippines. Jpn J Infect Dis. 2003;56:26-8. 
6. Chung MH, Lee SH, Kim MJ, Lee JH, Kim ES, Lee JS, et al. Japanese spotted fever, South Korea. Emerg Infect Dis. 2006;12:1122-4. https://doi.org/10.3201/eid1207.051372

7. Gaywee J, Sunyakumthorn P, Rodkvamtook W, Ruang-areerate T, Mason CJ, Sirisopana N. Human infection with Rickettsia sp. related to R. japonica, Thailand. Emerg Infect Dis. 2007;13:657-9. https://doi.org/10.3201/eid1304.060585

8. Ando S, Fujita H. Diversity between spotted fever group Rickettsia and ticks as vector. Jap J Sanit Zool. 2013;64:5-7. https://doi.org/ 10.7601/mez.64.5

9. Fournier PE, Fujita H, Takada N, Raoult D. Genetic identification of rickettsiae isolated from ticks in Japan. J Clin Microbiol. 2002;40:2176-81. https://doi.org/10.1128/JCM.40.6.2176-2181.2002

10. Lu M, Tian JH, Yu B, Guo WP, Holmes EC, Zhang YZ. Extensive diversity of rickettsiales bacteria in ticks from Wuhan, China. Ticks Tick Borne Dis. 2017;8:574-80. https://doi.org/10.1016/ j.ttbdis.2017.03.006

11. Feng HM, Chen TS, Lin BH, Lin YZ, Wang PF, Su QH, et al. Serologic survey of spotted fever group rickettsiosis on Hainan Island of China. Microbiol Immunol. 1991;35:687-94. https://doi.org/10.1111/j.1348-0421.1991.tb01602.x
12. Li J, Hu W, Wu T, Li HB, Hu W, Sun Y, et al. Japanese spotted fever in eastern China, 2013. Emerg Infect Dis. 2018;24:2107-9. https://doi.org/10.3201/eid2411.170264

13. Lu Q, Yu J, Yu L, Zhang Y, Chen Y, Lin M, et al. Rickettsia japonica infections in humans, Zhejiang Province, China, 2015. Emerg Infect Dis. 2018;24:2077-9. https://doi.org/10.3201/ eid2411.170044

14. Li H, Zhang PH, Huang Y, Du J, Cui N, Yang ZD, et al. Isolation and identification of Rickettsia raoultii and in human cases: a surveillance study in 3 medical centers in China. Clin Infect Dis. 2018;66:1109-15. https://doi.org/10.1093/cid/ cix917

15. Chen Z, Yang X, Bu F, Yang X, Yang X, Liu J. Ticks (Acari: Ixodoidea: Argasidae, Ixodidae) of China. Exp Appl Acarol. 2010;51:393-404. https://doi.org/10.1007/s10493-010-9335-2

Address for correspondence: Hao Li and Wei Liu, Beijing Institute of Microbiology and Epidemiology, State Key Laboratory of Pathogen and Biosecurity, 20 Dong-Da St, Fengtai District, Beijing 100071, China; email: lihao_1986@126.com and liuwei@bmi.ac.cn

\section{The Public Health Image Library (PHIL)}
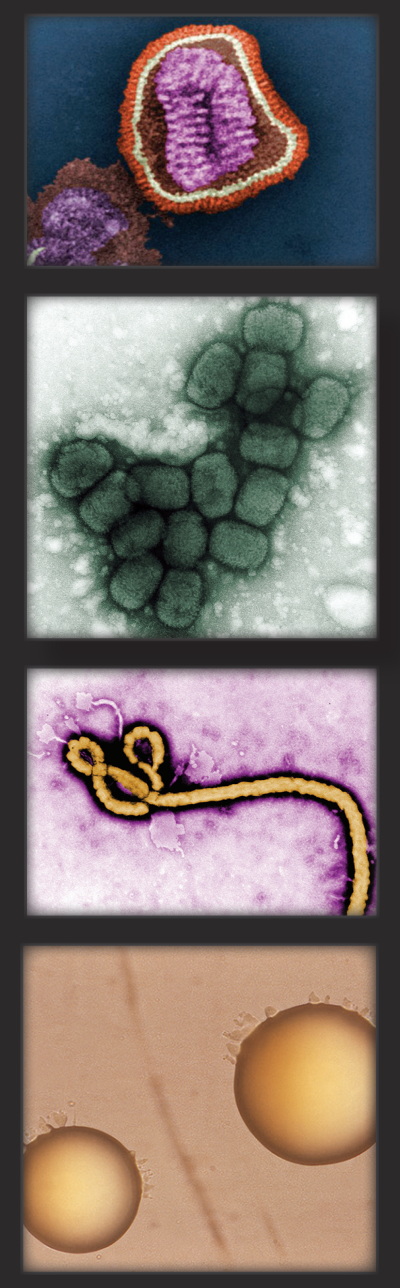

The Public Health Image Library (PHIL), Centers for Disease Control and Prevention, contains thousands of public health-related images, including high-resolution (print quality)photographs, illustrations, and videos.

PHIL collections illustrate current events and articles, supply visual content for health promotion brochures, document the effects of disease, and enhance instructional media.

PHIL images, accessible to PC and Macintosh users, are in the public domain and available without charge.

Visit PHIL at: http: / /phil.cdc.gov/phil 